\title{
Wort - Bedeutung - Begriff: Relationen und ihre Geschichte
}

\author{
Gerhard Strauß
}

\section{Teil I: Paradigmen im historischen Wandel}

Im folgenden sollen Aspekte der Relation zwischen den theoretischen Kategorien WORT, BEDEUTUNG und BEGRIFF herausgearbeitet werden, die in der Sprachwissenschaft vom späten 18. Jahrhundert bis in die erste Hälfte des 20 . Jahrhunderts eine Rolle gespielt haben und die auch heute noch diskutiert werden. Dies geschieht keineswegs in exhaustiver Weise, sondern ich will nur einige wesentliche Grundlinien und Wendepunkte in der Geschichte des Verhältnisses zwischen diesen Größen aufzeigen. Ich verfahre auch nicht strikt historisch-chronologisch, weil nicht immer inhaltlich zusammengehörige Ansätze auch chronologisch benachbart sind, also mit Diskontinuitäten zu rechnen ist, und weil schon bei einzelnen Autoren diskontinuierliche Perspektivenwechsel auftreten. Vielmehr will ich von einer klassifizierenden Schwerpunktsetzung ausgehen, die sich aus dem Zusammenhang mit dem derzeitigen theoretischen Diskussionsstand ergibt. Die hier relevanten Aspekte können mit Hilfe eines (im Anhang aufgeführten) Rasters herausgestellt werden, das - auch aus heutiger Sicht - wichtige Parameter für die Analyse einer Bedeutungskonzeption bereitstellt. Solche miteinander vernetzten Parameter sind zum Beispiel der Aspekt der Monolateralität gegenüber der Bilateralität des Wort- beziehungsweise Zeichenbegriffs, die Bezogenheit von Bedeutungen auf Begriffe beziehungsweise Konzepte, die Fassung von BEGRIFF selbst (etwa im Rahmen einer idealistischen, realistischen versus nominalistischen Begriffskonzeption), die Unterscheidbarkeit von enzyklopädischem beziehungsweise konzeptuellem und lexikalischem Wissen, und so weiter.

Dieses Raster werde ich hier nicht in ganzer Breite vorstellen, sondern mich nur auf einige Punkte beziehen. Aus einer solchen 'parametrisierten' Beschreibung historischer semantischer Positionen lassen sich Parallelen oder Divergenzen zur Moderne ableiten - unter Moderne verstehe ich vor allem das Paradigma des Strukturalismus, einschließlich poststrukturalistischer Fortführungen (vor allem im Sinne der Gebrauchstheorie der Bedeutung; vergleiche Teil II sowie die Beiträge von Keller und Harras in diesem Band), und das Paradigma des Kognitivismus. Dabei sind Merkmale oder Wesenszüge zu erkennen, die in 
modernen Positionen (in veränderter Form) wieder aufgegriffen werden (zum Beispiel die Re-Importierung der Kategorie BEGRIFF im moderneren kognitivpsychologischen Sinn von KONZEPT, einer Kategorie, die auch bereits im 18./19. Jahrhundert in unterschiedlichen Bedeutungstheorien eine wichtige Rolle spielt). Um diese Bezüge zu verdeutlichen, werden tentativ fünf Paradigmen eingeführt, wobei die Paradigmenbezeichnungen jeweils als Kurznamen für spezifische Parameterkombinationen aufgefaßt werden sollen.

\section{Das klassisch-dyadische Paradigma}

Das erste Paradigma nenne ich das klassisch-dyadische Paradigma und meine damit, daß in diesem Paradigma nur zwei Größen eine Rolle spielen, und zwar einerseits das Wort, hier eingeengt auf den Wortkörper (Signifikanten), und andererseits der als sprachunabhängig verstandene Begriff.

Diese erste Schwerpunktsetzung umfaßt die Aufklärungslexikographen des ausgehenden 18. Jahrhunderts sowie den Beginn der theoretischen Semasiologie und Onomasiologie im 19. Jahrhundert. Kennzeichnend ist ein monolateraler Zeichenbegriff, bei dem nur die Ausdrucksseite (also die Lautform) als sprachlich betrachtet wird und die 'Bedeutung' ins außersprachliche Reich der Vorstellungen oder Begriffe fällt, wo also die tradierte (klassische) dyadische Vorstellung von der Relation zwischen Wort und Begriff vorliegt.

Für Johann Christoph Adelung macht die "Verbindung der Vorstellungen mit den Worten oder Zeichen ihre Bedeutung " aus (Adelung, 1789, S. 125). Das Wort wird als materielles Zeichen betrachtet, das für eine Vorstellung oder einen Begriff steht, die in Verbindung mit einem Wort den Terminus "Bedeutung" erhalten. Dabei verweisen die Ausdrücke "Empfindung", "Vorstellung", "Begriff", die unter dem Terminus "Bedeutung" zusammengefaßt werden, auf diese extralinguale Konstituierung von Bedeutung. Trotzdem finden sich in Adelungs Grammatisch-kritischem Wörterbuche der Hochdeutschen Mundart (2. Auflage 1796) - ähnlich auch in den Wörterbüchern von J. A. Eberhard und J. H. Campe - differenzierte, modern anmutende 'Bedeutungs'-Beschreibungen, die dem Aristotelischen Prinzip der Begriffsanalyse folgen (indem sie mit der Angabe von genus proximum und differentia specifica operieren). Diese $\gg$ Definitionen im Sinne rationaler Begriffsklärungen « (von Polenz, 1994, S. 190) können auch als strukturalistische Merkmalsbeschreibungen reinterpretiert werden.

Mit der lexikographischen Beschreibungspraxis beziehungsweise mit der semantischen Explikation bei Adelung werde ich mich in Teil II meiner Ausführungen näher befassen.

Auch der Begründer der theoretischen Semasiologie Christian Karl Reisig - wie in modifizierter Form seine Nachfolger Friedrich Haase und E. G. F. Heerdegen, die Weisgerber (1927, S. 55) zur "logisch-begrifflichen Richtung « der Bedeutungslehre zählt - vertritt eine monolaterale Zeichenkonzeption: 
Wörter dienen der 'Veräußerlichung' von vorsprachlichen Gedanken und Empfindungen. Sie sind den Begriffen nachgeordnet. Die Bedeutung eines Wortes ist im Sinne Reisigs als Repräsentation einer sprachunabhängigen gedanklichen Größe durch einen materiellen Signifikanten zu bestimmen (vergleiche Schmitter, 1987, S. 117f.). Sprache ist somit nur Nomenklatur für autonome Vorstellungen und Gedanken. Ähnliche Auffassungen begegnen bei Heerdegen und bei Haase (1874-80, S. 71): Die Bedeutung ist ein Begriff, insofern dieser mit einem Worte als seinem Zeichen oder Abbilde verbunden ist. Dabei ist Reisig, wie die meisten Wissenschaftler seiner Zeit, insbesondere was die Beziehung zwischen Begriff und Welt angeht, einem Idealismus Kantscher Prägung verpflichtet (zu Kants Einfluß vergleiche Schmitter, 1987, S. 119 und 151).

Diese Position ist dadurch gekennzeichnet, daß angenommen wird, das menschliche Erkenntnissystem erst erschließe die bewußtseinsunabhängige Welt, die als solche nicht erkennbar ist. Die Erkenntnis und damit die Konstitution von Begriffen ist geprägt durch die transzendentalen Anschauungsformen Zeit und Raum sowie durch sogenannte Verstandeskategorien wie Quantität, Qualität, Relation und Modalität. Diese universalistisch-idealistische Begriffskonzeption Kantscher Prägung ist (in meinem Raster) zu kontrastieren einerseits mit einer realistischen, andererseits mit einer nominalistisch-individualpsychologischen Konzeption.

Ich möchte gleich an dieser Stelle diese beiden anderen Begriffsauffassungen kurz skizzieren: Erkenntnistheoretischer Realismus, bei dem angenommen wird, Sachverhalte könnten so erkannt werden, wie sie tatsächlich in der bewußtseinsunabhängigen Welt sind, führt zur Annahme von Allgemeinbegriffen als Abbildern objektiv gültiger Differenzierungen in der Welt. Diese Position spielt für die weitere Erörterung keine gewichtige Rolle mehr, man mag sie im amerikanischen Strukturalismus partiell wiedererkennen (vergleiche Abschnitt 4.2).

Im Nominalismus dagegen existieren keine überindividuellen Begriffe, sondern nur individuelle Vorstellungen. Wörter als Träger individueller Vorstellungen benennen nur mit willkürlichen Namen (Etiketten) die im Bewußtsein des Subjekts prinzipiell verborgenen Vorstellungen. Diese Position wird uns zum Beispiel bei Hermann Paul (im dritten Paradigma) noch begegnen.

Zurück zum Idealismus der frühen Lexikographen. Es ist klar, daß in dieser Position ebenso wie generell bei allen monolateralen Zeichenkonzeptionen eine Trennung zwischen lexikalischem Wissen und Weltwissen nicht angesetzt ist. Die Begriffe konstituieren sich ja unabhängig von Wörtern nur im Hinblick auf das verstandesmäßige Kategorisieren der Welt. Die 'Lexikalisierung' von Begriffen stellt dann einen Prozeß der - möglicherweise selektiven - Begriffsbenennung dar, eine eigene Ebene von 'Wortbegriffen' ist nicht vorzusehen: Bei diesem Zugang spricht nichts dagegen, alles enzyklopädische Wissen zum Beispiel über Hunde, Vögel, Früchte begrifflich anzubinden an die entsprechenden Wortkörper. Genau dies kritisiert zum Beispiel Hermann Paul $(1895$, S. 65) an den Bedeutungs- beziehungsweise Begriffserklärungen von naturwissenschaftli- 
chen Termini in den Wörterbüchern seiner Zeit: »Die Bedeutung von Tier- und Pflanzenbezeichnungen erklärt man wohl in den Wörterbüchern durch naturwissenschaftliche Definitionen mit Angabe der Klasse etc. Nehmen wir aber z. B. eine solche zoologisch ganz korrekte Definition von "Hund", so sind wir weit entfernt davon, damit diejenige Vorstellung von einem Hunde zu haben, die in dem allgemeinen Volksbewusstsein lebt, und diese ist es doch, welche die Bedeutung des Wortes ausmacht.«

Der universalistisch-anthropologische Idealismus dieser Position entspricht in der Grundtendenz dem modernen Kognitivismus, auch wenn natürlich jede empirische Kenntnis über die Struktur des menschlichen Gehirns/Geistes noch fehlt, insbesondere über die semantische Komponente unserer Sprachfähigkeit als eines im Gedächtnis gespeicherten Kenntnissystems.

\section{Das vorstrukturalistische Paradigma}

Ich komme nun zum zweiten Paradigma, das ich als vorstrukturalistisches Paradigma bezeichne: Die Gegenströmung zum begriffsorientierten Monolateralismus ist an Wilhelm von Humboldt festzumachen. Er kann als der große Anreger in Richtung eines bilateralen Zeichenbegriffs verstanden werden.

Humboldt nimmt an, den Einzelsprachen wohne ein gestaltendes Prinzip inne, das sowohl die lautliche äußere Form von Zeichen präge als auch die inhaltliche 'innere' Form. Er nimmt also an, erst die Sprache bilde zeichengebundene Begriffe aus, und zwar auf der Grundlage noch ungeformter sinnlicher Eindrücke und Geistesbewegungen. Humboldt beschreibt diesen 'Stoff, aus dem Bedeutungen gemacht werden, als »die Gesamtheit der sinnlichen Eindrücke und selbstthätigen Geistesbewegungen, welche der Bildung des Begriffs mit Hilfe der Sprache vorausgehen " (Humboldt, Akademie-Ausgabe VII, S. 49, vergleiche Schmitter, 1987, S. 52). Das gestaltende Prinzip, forma formans einer Einzelsprache, setzt also auf der lautlichen wie auf der begrifflichen Seite eine noch ungeformte stoffliche Substanz voraus, die jedoch - da dann bereits geformt - innerhalb der Sprache nicht mehr vorhanden und zugänglich ist. Formung findet dabei bereits auf beiden Seiten, auf der lautlichen wie auf der inhaltlichen für sich statt, zur Vollendung kommt das gestaltende Prinzip jedoch erst in der unauflöslichen Bindung einer äußeren (Sprach-) Form an eine innere (Sprach-) Form, also zum Beispiel in der "Articulation « eines bestimmten Wortzeichens. Diese sogenannte »Synthesis der äußeren und inneren Sprachform « (Humboldt, a. a. O., S. 96) schafft nämlich in einer Art qualitativem Sprung ein neues Ganzes, »das in keinem der verbundenen Theile für sich liegt « (S. 94).

Sprachliche Inhalte werden erst durch die Bindung an sinnlich wahrnehmbare Lautformen ausgegliedert: "Dadurch also, da $\beta$ das Wort als ertönendes, hervorgerufenes (und wieder vernommenes) den ansonsten unbestimmten Begriff in einen sinnlichen Stoff vor der Einbildungskraft verwandelt, 'schiebt (es) 
der Idee eine Gestalt unter' (Humboldt, Akademie-Ausgabe V, S. 428), wird es zum 'bildenden Organ des Gedankens' "(Jäger, 1987, S. 186). Diese artikulierten Inhalte konstituieren zum Beispiel als Wörter einer Einzelsprache "wahre Individuen «, die nur innerhalb der Sprache selbst, "wie es dem Sprachgebrauche der Wörter gemäß ist «, begrenzt werden.

In meinem Raster ist diese Position als einzelsprachlicher, nicht-universaler Idealismus einzuordnen: Die begriffliche Strukturierung der Welt ist gebunden an die begriffsprägenden Formen einer Einzelsprache, die als apriorische Erkenntnisform verstanden wird. Zu denken ist hier an Stichwörter wie "sprachliche Relativitätstheorie" oder "sprachliche Zwischenwelt" (Weisgerber).

Von der Position Humboldts aus ergibt sich eine klare Entwicklungslinie zu Ferdinand de Saussure mit seinem bilateralen Zeichenbegriff und so weiter und partiell auch zum Strukturalismus. Von hier aus leitet sich - wie gesagt - auch die Tradition des einzelsprachbezogenen Idealismus ab, mit einer Entwicklungslinie zu Weisgerber und der Sprachinhaltsforschung sowie der Wortfeldtheorie.

Andere Anreger für strukturalistische Gedanken und Vorgehensweisen sind zu erwähnen: K. W. L. Heyse und Carl Abel, die vor allem den Wortfeldgedanken mit ihren Arbeiten (in der zweiten Hälfte des 19. Jahrhunderts) vorgeprägt haben: Heyse in seinem System der Sprachwissenschaft (1856) und Abel in seinen Oxforder Vorlesungen (1882) sowie in der Abhandlung Die englischen Verba des Befehls (1878) (vergleiche Lutzeier, 1995, S. 10f.).

Nur bedingt dem vorstrukturalistischen Rahmen zuzuordnen ist die sogenannte Wörter-und-Sachen-Forschung, eine spezielle Richtung innerhalb der isolierten Einzelwortforschung zu Beginn des 20. Jahrhunderts. Sie stellt eine Art Sonderlinie innerhalb der vorstrukturalistischen Semasiologie und Onomasiologie dar, insofern sie - bei ihrer Konzentration auf Sprachgeschichte als Teil der Kultur- und Sachgeschichte - auf die Vermittlungsinstanz "Begriff" weitgehend verzichtet. In ihrer einfachsten Version arbeitet diese Richtung mit einem monolateralen Sprachzeichenbegriff, weil allein das Wort als lautlicher Ausdruck einer Sache, also innerlingual verstanden wird, und die Sache, als etwas Extralinguales, weitgehend als die Bedeutung des Wortes aufgefaßt wird (Wiegand \& Harras, 1971, S. 94). Das heißt, die Bedeutung wird durch eine Analyse der Sache gewonnen und meist unreflektiert mit dieser gleichgesetzt; dabei meint der 'Bedeutungs'-Begriff jedenfalls weder den Wortinhalt (verstanden als Teil des Wortes oder, wie Weisgerber, 1927, S. 61 sagt, als die "Bedeutung im Worte «) noch die Beziehung zwischen Wort und Sache.

Dem monolateralen Zeichenbegriff entspricht ein zweipoliges Zeichenschema, in dessen Zentrum die einfach benennende Zuordnung eines Ausdrucks und einer Sache steht. Mit "Sache" wird in einem weiten Sinn alles Außersprachliche gefaßt, das Sinnliches wie Unsinnliches einschließt. Dabei wird jedoch nicht hinterfragt, ob mit "Sache" der jeweils einzelne Referent (der parole) gemeint ist oder eine abstrakte, virtuelle Einheit (der langue), da eine Unterscheidung zwischen langue und parole nicht gemacht wird. Neben unmittelbaren 
Zuordnungsbeziehungen wie »das Wort steht für eine sinnlich wahrnehmbare Sache « oder (bei Nicht-Konkreta) »für eine Vorstellung" gibt es bei Schuchardt theoretisch auch differenziertere Aussagen, indem - unter Berufung auf das Scholastische voces significant res mediantibus conceptibus - eine vermittelnde Instanz eingeführt wird: Das Wort steht für eine sinnlich wahrnehmbare Sache vermittels der Vorstellung von dieser Sache, die Sachen hängen also nur vermittels der Vorstellungen mit den Wörtern zusammen (vergleiche Schuchardt, 1912, S. 830 und 832).

Der Schwerpunkt des wissenschaftlichen Interesses liegt auf der Geschichte der 'Sachen' und auf dem Wandel ihrer Bezeichnungen. Bedeutungswandel wird daher als Sachwandel interpretiert, Wortbedeutung und Sache werden, bezogen auf einen synchronen Sprachzustand, weitgehend miteinander identifiziert. Zwar wird zwischen Wort und Bezeichnung, Bedeutung und Sache zunächst methodisch unterschieden, es werden also jeweils vier (beziehungsweise mit der 'Vorstellung' fünf) verschiedene Größen unterschieden. Es wird jedoch - gemäß Schuchardts Auffassung, derzufolge Sprachgeschichte ein Fortschreiten von und zu angenommenen 'Ruhepunkten' ist - postuliert, daß sich in einem solchen 'Ruhepunkt' als synchronem Schnitt innerhalb der Diachronie Wort und Bezeichnung (für die Sache) einerseits, Bedeutung und Sache andererseits jeweils 'decken' können: ". . in jedem Ruhepunkt deckt sich eine Bezeichnung mit einem Wort und eine Bedeutung mit einer Sache " (Schuchardt, 1912, S. 832); nur in historischer Perspektive können sich die Paare (Wort Bezeichnung/Bedeutung - Sache) getrennt voneinander entwickeln. In den 'Ruhepunkten' zeigen sich zumindest Ansätze oder meine besondere Art von bilateraler Sprachzeichenauffassung « (Wiegand \& Harras, 1971, S. 98; vergleiche auch Weisgerber, 1927, S. 76), die sich zum Beispiel unter dem Aspekt der individuellen Genese einer Wortbedeutung wie folgt beschreiben läßt: Eine Sache (S) wird von einem Sprecher wahrgenommen, es entsteht in dessen Bewußtsein eine Vorstellung (V) von dieser Sache, aufgrund dieser Vorstellung kann (S) bezeichnet werden und zwar mit einem Wort (W), diesem Wort (W) wird durch den Bezeichnungsakt eine Bedeutung (Bd) zugeordnet, die sich mit (S) deckt. Insgesamt entsteht eine bilaterale Bezeichnung (Be), in der sich die Sache (S) ausdrückt (Wiegand \& Harras, 1971, S. 98 und S. 108ff. mit der Angabe von Wortbeispielen).

Die Verfahrensweise der Wörter-und-Sachen-Forschung kann insgesamt als onomasiologisch beschrieben werden insofern, als der Analyseausgangspunkt etwas Außersprachliches ist, aber auch als semasiologisch insofern, als das sprachwissenschaftliche Interesse auf die 'Wortbedeutung' abzielt und damit zugleich auf die 'Bezeichnung' als Einheit von 'Wort' und 'Bedeutung' (Wiegand \& Harras, 1971, S. 100). Beide Einstufungen gelten jedoch nur mit Einschränkungen, da eine konsequente Vermittlungsinstanz "Begriff" beziehungsweise "Wortinhalt" nicht angenommen wird. 


\section{Das vorkognitivistische oder psychologische Paradigma}

Das dritte Paradigma bezeichne ich als vorkognitivistisches oder psychologisches Paradigma: Während vom vorstrukturalistischen Paradigma eine Entwicklungslinie zum Strukturalismus führt, ist aus der semantischen Position Hermann Pauls, für die der Einfluß völkerpsychologischer Theorien wichtig ist, eine Verbindungslinie zum Kognitivismus abzuleiten. Paul wird hier als prominentester Vertreter einer individual-psychologischen Bedeutungstheorie ausführlicher behandelt; daneben sind zu nennen M. Hecht ("Bedeutungen sind mit den Worten verbundene Vorstellungen«), W. Wundt (»Bedeutungen sind psychologische Begriffe $«$ ), E. Wellander ( $»$ Die Bedeutung eines Wortes ist die Vorstellung, die ein Individuum mit diesem Worte verbindet «) und A. Marty ("Die Bedeutung ist dasjenige psychische Phänomen, welches der sprachliche Ausdruck im Hörer wachzurufen bestimmt ist «), die von Weisgerber (1927, S. 59) unter der »psychologisch-erklärenden Richtung « der Semasiologie zusammengefaßt werden.

Nach Paul ist Sprache im eigentlichen Sinne auf das private unzugängliche Innenleben des Individuums beschränkt. Nur die Individualsprache hat Realität: Das einzig "wahre Objekt für den Sprachforscher sind . . . sämtliche Äußerungen der Sprechtätigkeit an sämtlichen Individuen in ihrer Wechselwirkung aufeinander « (Paul, 1880, S. 24). 'Usus' und 'Gemeinsprache' erscheinen ihm als nützliche Abstraktionen. Sprache und Bedeutung sind also an das psychische Innenleben des einzelnen gebunden; Bedeutungen konstituieren sich nach Pauls Auffassung als Vorstellungen von Individuen (»psychischen Organismen «; Paul, 1880, S. 28), die "gruppenweise «, also als Vorstellungskomplex(e), auf der Basis von Gehörtem oder Gesprochenem ins Bewußtsein eingeführt werden. Paul ist somit ein Vertreter einer nominalistischen Bedeutungsauffassung. Er bekennt sich ausdrücklich zu der nominalistischen Grundauffassung, Vorstellungsinhalte seien nicht übertragbar; in seinen Prinzipien (1880, S. 15) heißt es: "Der Vorstellungsinhalt selbst ist also unübertragbar. Alles was wir von dem eines andern Individuums zu wissen glauben, beruht nur auf Schlüssen aus unserem eigenen.« Zwar kann ein Individuum durch das von ihm erzeugte physische Produkt (Lautgebilde) auf dem Wege der "Erregung der sensitiven Nerven « in der Seele »eines anderen Individuums entsprechende Vorstellungen hervorrufen (Paul, 1880, S. 14). Da jedoch die Beziehung zwischen dem Sprachlaut und der erzeugten Vorstellung nur eine indirekte, durch Ideenassoziation vermittelte ist, ist keineswegs ausgemacht, daß in den verschiedenen Seelen die gleiche Assoziation stattfindet. Wie eine solche Angleichung bewerkstelligt werden kann, erklärt Paul nur unbefriedigend durch die Annahme »übereinstimmender Erfahrung « beziehungsweise dadurch, daß wir in der Lage seien, "indirekte Assoziationen in direkte « zu verwandeln. Das bereits von John Locke (vergleiche Trabant, 1976, S. 25f.) dargelegte grundsätzliche Dilemma des Nominalismus "Wie ist Kommunikation bei unzugänglichen Innenwelten überhaupt möglich?" wird auch bei Paul nur scheinbar gelöst. 
Pauls Standpunkt eines mentalistischen Individualismus führt also zu dem bekannten Problem des Nominalismus im Hinblick auf die Möglichkeiten von Kommunikation und Verständigung überhaupt. Insofern ist Pauls Position verschieden vom universalistisch geprägten Kognitivismus neuerer Provenienz, bei dem eine gemeinsame mentale Grundausstattung der Individuen die Voraussetzung für ähnlich strukturierte Innenwelten und damit für die Austauschbarkeit von Erfahrungs- und Bewußtseinsinhalten schafft.

Sieht man davon ab, ergeben sich jedoch klare Parallelen zwischen Pauls mentalistischer Bedeutungskonzeption und kognitiven Ansätzen: Paul unterscheidet zwischen usueller Bedeutung, das ist der gesamte Vorstellungsinhalt, den ein Sprecher mit einem Wort verbindet, und okkasioneller Bedeutung, das ist derjenige Vorstellungsinhalt, welchen der Redende, indem er ein Wort ausspricht, mit diesem verbindet und von dem er erwartet, daß ihn auch ein Hörer damit verbinde. Die okkasionelle Bedeutung, so sagt Paul, ist gewöhnlich an Inhalt reicher, an Umfang enger als die usuelle: ersteres, weil die okkasionelle Bedeutung einen konkreten, durch räumliche und zeitliche Verankerung angereicherten Vorstellungsinhalt repräsentiert, letzteres, weil die okkasionelle Bedeutung gegenüber der usuellen selektiv ist, indem sie eine Auswahl aus dem gesamten, mit der usuellen Bedeutung assoziierten Vorstellungsinhalt darstellt. Paul beschreibt den Übergang von usueller zu okkasioneller Bedeutung bei der Sprachproduktion als ein Kontextualisierungs- und Individualisierungsphänomen, das einerseits bei polysemen Wörtern die Disambiguierung von Lesarten ermöglicht. Denn okkasionell ist die Bedeutung eines Wortes immer »einfach «, auch dann, wenn das Wort selbst eine "mehrfache " usuelle Bedeutung hat. Andererseits geschieht in diesem Prozeß auch der Übergang beispielsweise von Gattungsbegriffen zu Individualbegriffen mit definiter Referenz (zum Beispiel "Baum" $\rightarrow$ "dieser bestimmte Baum"). Kontextualisierung von Nomina erfolgt durch den Gebrauch des Artikels, eventuell durch Bestimmungswörter in Komposita (zum Beispiel "Nadel": "Stopfnadel", "Nähnadel"), durch Situationsbezug ("gemeinsame Anschauung" in der Redeweise Pauls), durch Textreferenz und gemeinsamen Erfahrungshintergrund. So beschreibt Paul (1880, S. 79) das Phänomen, mit Angaben wie "das Rathaus", "der Vater", "die Dame" auf rein sprachlich nur unzureichend eingegrenzte Objekte definit Bezug nehmen zu können: Hier sichert erst die gemeinsame Orientierung auf das herausragende ("saliente «), am ehesten oder alleine in Frage kommende Objekt dieser Art die Verständigung.

Meines Erachtens gibt es hier sehr starke Parallelen zum modernen Kognitivismus. Die Unterscheidung zwischen usueller Bedeutung und okkasioneller Bedeutung präfiguriert die kognitivistische Unterscheidung zwischen im Langzeitgedächtnis gespeichertem Konzept und aufgrund von Kontext und Situationswissen erzeugter aktueller Bedeutung beziehungsweise Äußerungsbedeutung als Einheit des Kurzzeitgedächtnisses im Sprachverarbeitungsprozeß. Die kognitive Semantik beschreibt ganz analoge Kontextualisierungsphänomene wie Paul als Bedeutungseingrenzungsphänomene. Die aktuelle Bedeutung ist wie 
bei Paul eine Vorstellung, eine mentale Repräsentation, die den Zugriff auf ein konkretes Gemeintes (den Referenten) ermöglicht.

Schwarz (1992, S. 129) nennt aus der Sicht der kognitiven Semantik unter anderem folgende Schritte der Bedeutungsspezifizierung:

(a) die Selektion einer Bedeutungslesart bei mehrdeutigen Wörtern;

(b) die Spezifizierung einer Bedeutung;

(c) die Hervorhebung bestimmter Bedeutungsmerkmale;

(d) die Verschiebung typischer Bedeutungsmerkmale beziehungsweise Instanzen.

Alle diese Schritte sind bei Paul zumindest angedeutet, auf Lesartenselektion und Spezifizierung (am Beispiel "Nadel": "Näh-", "Stopfnadel") habe ich bereits hingewiesen. (c) und (d) erscheinen bei Paul unter dem Stichwort "Abweichung" in dem Sinne, daß die okkasionelle Bedeutung nicht alle Elemente der usuellen einschließt (vergleiche Paul, 1880, S. 82). Paul konstatiert als allgemeine Grundbedingung für die Möglichkeit einer solchen »bloß partiellen Benutzung " der usuellen Bedeutung eines Wortes, daß diese aus mehreren Elementen besteht (a. a. O., S. 82). Er vertritt also eine Art dekompositionale Bedeutungsauffassung. Die Formen, die Paul in diesem Zusammenhang nennt, sind unterschiedliche Ausprägungen bildlichen, metaphorischen, metonymischen Sprachgebrauchs, wobei ihn insbesondere der Übergang von der individuellen Ausdehnung zu echtem Bedeutungswandel interessiert. Bemerkenswert ist, daß Paul dabei auch das Phänomen der Konzeptverschiebung (im Sinne von Bierwisch, 1983) erfaßt, wenn es heißt: »Andererseits werden "Ministerium", "Amt", "Gericht", "Universität" etc. zu Bezeichnungen der Gebäude, in denen sie [gemeint sind die betreffenden Institutionen beziehungsweise die dort Beschäftigten, G. S.] ihren Sitz haben “ (Paul, 1880, S. 98).

Ein wesentlicher Gesichtspunkt im Vergleich zwischen mentalistischen und strukturalistischen Bedeutungsauffassungen ist, daß sich die okkasionelle/aktuelle Bedeutung, die sich ja auf ein konkretes Referenzobjekt beziehungsweise eine konkrete Referenzsituation richtet, weitgehend von der usuellen Bedeutung ablösen kann. Die aktuelle Bedeutung zum Beispiel von "Vogel" kann dann letztlich der Zugriff auf ein bestimmtes Huhn sein (vergleiche Schwarz, 1992, S. 129). Ähnlich läßt auch Paul schon zu, daß die individuelle Bedeutung "nur auf eine von den verschiedenen Arten geht, die in dem generellen Begriffe enthalten sind (Paul, 1880, S. 78). Dies widerspricht der strukturalistischen Bedeutungskonzeption. Denn nach strukturalistischer Auffassung ändert sich die Bedeutung von "Vogel" nicht, auf welches Element der Kategorie VOGEL man auch referiert. Ob ich mit "dieser Vogel" ein Huhn oder einen Kolibri meine, ich habe allemal nur gesagt, daß es sich bei dem Gemeinten um einen Vogel handelt. Das identische Gesagte hat in beiden Fällen selbstverständlich auch dieselbe Bedeutung. Daß ich mit "dieser Vogel" immer nur bestimmte Exemplare, die ich dann auch als "dieses Huhn"/"dieser Kolibri" bezeichnen 
könnte, meinen kann, ist aus strukturalistischer Sicht kein Bedeutungs(eingrenzungs)phänomen, sondern resultiert aus dem Hyperonym-Hyponym-Verhältnis zwischen "Vogel" und "Huhn"/"Kolibri".

Gerade an dieser unterschiedlichen Interpretation des Verhältnisses zwischen der Bedeutung eines Zeichens und der Bedeutung der konkreten Verwendung dieses Zeichens wird der große Unterschied zwischen der Auffassung von Sprache als System und der Paulschen beziehungsweise kognitiven Auffassung von Sprache als Sprachfähigkeit und Sprachverarbeitung deutlich. Es konnte insbesondere herausgearbeitet werden, daß und inwiefern Pauls Unterscheidung zwischen usueller und okkasioneller Bedeutung die Unterscheidung zwischen (im Langzeitgedächtnis gespeichertem) Konzept und aufgrund von Situationswissen erzeugter aktueller Bedeutung präfiguriert, daß aber, wenn $\mathrm{Be}$ deutungen - wie im kognitiven Paradigma - als mentale Einheiten begriffen werden, die Trennung zwischen den beiden Größen Bedeutung und Gemeintem beziehungsweise $\ddot{A} u ß e r u n g s b e d e u t u n g$ tendenziell aufgehoben wird.

$\mathrm{Zu}$ ergänzen sind einige Anmerkungen zu Pauls theoretischen Vorstellungen zur Lexikographie, soweit sie mit seiner mentalistischen Bedeutungsauffassung im Zusammenhang stehen. Völlig im Einklang mit dieser Bedeutungstheorie steht seine Auffassung, Aufgabe des Lexikographen sei es, den Vorstellungsinhalt, der die Bedeutung eines Wortes ausmache, in all seinen einzelnen Momenten auf den Leser zu übertragen (Paul, 1895, S. 64). Dies, so Pauls kritische Anmerkung, die er auch gegen Adelung richtet, könne nicht in allen Fällen in Form einer Aristotelischen Definition geschehen. Denn es gebe eine Reihe von Wörtern, bei denen jede Definition versagen müsse, weil sie eine »einfache Vorstellung" ausdrückten, wie etwa die Farbwörter "blau", "rot" und so weiter (Paul, 1895, S. 65). Bei diesen Wörtern habe die sinnliche Anschauung allein die entsprechende Vorstellungsassoziation bewirkt. Insofern beschränke sich die Aufgabe des Lexikographen, der Wörter nur durch Wörter beschreiben könne, sinnvollerweise darauf, mit den bereits in der Seele des Lesers erzeugten und an bestimmte Wörter assoziierten Vorstellungskomplexen in zweckmäßiger Weise zu operieren. Konsequenterweise empfiehlt Paul daher, die »normalen « Bedeutungen von Wörtern wie "rot", "süß", "Affe", "Hand", "schreiben" (a. a. O., S. 67) einfach als bekannt vorauszusetzen. Auch in diesem Punkt ist Paul sicherlich moderner als etwa Adelung oder Heyse, weil er die Grenzen lexikographischer Definitionen, insbesondere die Gefahr der Zirkularität, erkennt, und insofern, als er auf die Sonderstellung von bestimmten einfachen Konzepten (zum Beispiel Farbwörter, natural kind-Wörter) hinweist, die in der neueren Forschung (zum Beispiel Blutner, 1995; Johnson-Laird, 1987) immer wieder herausgearbeitet wird.

Erwähnt werden soll hier im Zusammenhang mit dem Kognitivismus K. O. Erdmanns Bedeutungskonzeption, die er in seinem Buch Die Bedeutung des Wortes. Aufsätze aus dem Grenzgebiet der Sprachpsychologie und Logik (1. Auflage 1900) in interessanter und oft modern anmutender Weise darlegt. Erdmann versucht, die »logisch-klassifizierende Richtung (vergleiche oben das er- 
ste Paradigma) und die "psychologisch-erklärende Richtung « der Semasiologie miteinander zu verbinden, und gelangt zu seiner besonders bekannt gewordenen Dreiteilung der "allgemeinen Wortbedeutung «. Dabei wird der begriffliche Inhalt von größerer oder geringerer Bestimmtheit vom Nebensinn und vom $G e-$ fühlswert abgegrenzt. Diese Dreiteilung als interne Gliederung der Wortbedeutung könnte suggerieren, daß dem gesamten Modell ein einheitlicher Bedeutungsbegriff zugrunde liegt. Daher haben zahlreiche Linguisten unter Bedeutung nur den objektiven begrifflichen Inhalt verstanden und den Nebensinn der Wörter (zusammen mit dem Gefühlswert) in das Reich der Konnotationen verwiesen beziehungsweise als für die linguistische Semantik irrelevant aus ihren Untersuchungen ausgeklammert, wodurch sie gerade das eliminierten, was ihr eigentlicher Hauptgegenstand ist: die einzelsprachspezifischen Bedeutungen der Wörter und die einzelsprachspezifische Gliederung der Welt durch das Lexikon einer Sprache (vergleiche Keller in diesem Band). Dieser Verwirrung hat Erdmann selbst Vorschub geleistet, denn in Wirklichkeit liegen seinem Modell zwei verschiedene Bedeutungsbegriffe zugrunde, wie es der Untertitel seines Buches andeutet: ein logischer und ein psychologischer, die nicht einfach kombinierbar und in einem Schema addierbar sind (so Dieckmann, 1981, S. 95f.).

Während Erdmann der Analyse von Dieckmann zufolge mit seiner Unterscheidung zwischen begrifflichem Inhalt und Nebensinn der Existenz von extensionsgleichen, jedoch intensional (im 'Nebensinn') verschiedenen Wörtern wie "Säugling", "Wickelkind" und "Neugeborenes" Rechnung zu tragen suchte und damit in die Nähe der Fregeschen Unterscheidung zwischen Bedeutung und Sinn vorstieß, kann er mit der Differenzierung zwischen Bedeutungszentrum (auch "Kern" genannt) und Bedeutungsperipherie (auch "Grenzgebiet" genannt) als Vorläufer der Prototypentheorie betrachtet werden (vergleiche Blutner, 1995, S. 227f.). Nach seiner Auffassung sind die meisten Wörter einer natürlichen Sprache nicht klar bestimmt und scharf abgegrenzt, sondern "Worte sind vielmehr im allgemeinen Zeichen für ziemlich unbestimmte Komplexe von Vorstellungen, die in mehr oder minder loser Weise zusammenhängen. ... Die Grenzen der Wortbedeutung sind verwaschen, verschwommen, zerfließend « (Erdmann, 1910, S. 4f.). Dies zeigt er zunächst an alltagssprachlichen, einfachen Wörtern oder "Popularbegriffen « wie "Stuhl" oder "Tisch". Diesen stellt er zu wissenschaftlichen Zwecken gebildete Ausdrücke gegenüber, die »nur eine einzige, scharf begrenzte Bedeutung haben« (Erdmann, 1910, S. 33). Erdmann erkennt also den Unterschied zwischen Fregeschen Begriffen (mit der Eigenschaft der 'scharfen Begrenzung') und unscharfen Begriffen. Erstere werden (nach Keller, 1995, S. 87) durch Definitionen erzeugt, die ausschließlich Gegenstandsmerkmale - Erdmann nennt zum Beispiel anatomische und physiologische Merkmale bei der Klassifikation von Tieren - als Gebrauchskriterien benutzen. Sie »bilden einen verschwindenden Bruchteil aller Worte. Man könnte zu ihnen allenfalls Zahlworte rechnen und jene wissenschaftlichen Fachausdrücke, die in die Alltagssprache niemals eingehen, wie "Potential", 
"Elliptische Funktion", "Wismutoxyd" ...« (Erdmann, 1910, S. 33), ferner weisen nur die Mathematik und die exakten Naturwissenschaften ganz eindeutige, dem logischen Ideal entsprechende Termini auf, während bei den Termini der übrigen Wissenschaften »unklare Grenzgebiete (S. 34) unvermeidlich sind. Die Mehrzahl der Wörter beziehungsweise Wortbedeutungen einer natürlichen Sprache ist unscharfen Begriffen zugeordnet, bei denen wursprünglich ein breites, unklares Grenzgebiet vorhanden « (S. 30) ist; dies gilt natürlich auch für die Terminologien der Wissenschaften, die den "größten Teil ihrer Ausdrücke mit der Alltagssprache gemein « (S. 34) haben. Als Beispiele nennt er Ausdrücke, die in den Wissenschaften in einer spezialisierten, vom weiteren alltagssprachlichen Gebrauch abweichenden Bedeutung verwendet werden (zum Beispiel "Kreis" in der Mathematik, "Besitzer" in der Jurisprudenz).

Ganz besonders deutlich aber ist die Unmöglichkeit, feste Grenzlinien zu ziehen, "wenn ein Wort einem Begriff zugehört, der gradweise Abstufungen zuläßt und durch unmerkliche Übergänge in sein Gegenteil überführt werden kann. In solchen Fällen kann die Sprache der Wirklichkeit nicht gerecht werden: diese zeigt stetige Übergänge, während jene in den Worten nur diskrete Werte besitzt “ (S. 31). "Solche 'fließende' Grenzen finden sich oft « (S. 31), nicht nur bei den Farbwörtern (zum Beispiel bei Erdmann die Skala der Grautöne), sondern auch bei Popularwörtern wie "Mittelstand" (ab welcher Einkommensgrenze gehört man dazu?), "Haufen" (beim wievielten Korn beginnt er?) oder "Kind", "jugendliche Person", "Mannes-", "Greisenalter". Meist sind zu ihrer Bestimmung nicht ein einzelnes, sondern mehrere verschiedene, sich oft kreuzende Merkmale erforderlich, die den »Wortsinn schwanken« machen (S. 32). Ob zum Beispiel jemand ein Kind genannt wird, hängt nicht nur von der Zahl der Lebensjahre, sondern auch von gewissen körperlichen und geistigen Merkmalen der betreffenden Person ab. Wenn hier, zum Beispiel für juristische Zwecke, ein einziges Merkmal zum ausschlaggebenden gemacht und für dieses eine feste Grenzlinie gezogen werden muß, »dann ist es natürlich ganz unvermeidlich, daß in gewissen Fällen die der Definition folgende Kennzeichnung der Person dem natürlichen Sprachgebrauch ins Gesicht schlägt“ (S. 32). Auch die oben bereits erwähnten ganz einfachen und scheinbar wohlbestimmten Bezeichnungen für Artefakte wie "Tisch", "Stuhl", "Tasse" haben keine festen Grenzen: Jede Definition - so Erdmann - könne hier als zu weit oder zu eng angefochten werden. Damit erkennt Erdmann die extensionale Unbestimmtheit sprachlicher Ausdrücke als generelle Eigenart alltagssprachlicher Begriffe oder 'Popularbegriffe', im Gegensatz zu den logischen Begriffen. Zwar ist die Intension der alltagssprachlichen Ausdrücke eine Nominaldefinition (gespeist von lexikalischem Wissen), also ein Merkmalskomplex, der selbst nicht vage ist. Erst die Extensionalisierung führt zu Vagheit und Prototypizität (enzyklopädisches Wissen) (vergleiche Blutner, 1995, S. 251f.). Diese begriffliche Unbestimmtheit gelte auch für »jene zahllosen Alltagswörter«, die in juristischen Bestimmungen eine Rolle spielen, zum Beispiel "Angehöriger", "Dienstbote", "Geheimnis", "Gebäude", "bewegliche Sache", "bei Nacht". 
Mit dieser Wortgruppe sind wir beim zweiten hier interessierenden Aspekt, den Erdmann unter dem Kapitel Wortanalyse und Wortabgrenzung beziehungsweise -begrenzung behandelt: der Abhebung auf die lebensweltliche Kommunikationspraxis und die Rolle des alltagssprachlichen Wortschatzes in fachlichen Zusammenhängen, vor allem juristischen Texten. Während die Wortanalyse den Sprachgebrauch beschreibt, mithin richtig oder falsch sein kann, legt die Wortabgrenzung bestimmte Sprachgebräuche beziehungsweise die Bedeutungen bestimmter (auch ursprünglich alltagssprachlicher) Wortgebräuche fest; bei ihr stellt sich nicht die Frage nach wahr oder falsch, vielmehr ist die Bildung logisch vollkommener Begriffe, sind Bedeutungsfestlegungen zu beurteilen nach den Kriterien zweckmäßig/brauchbar oder nicht. Der Wert von Wortabgrenzungen hängt ab von den Bedürfnissen, denen sie dienen. So ist zum Beispiel die zoologisch 'falsche' Bezeichnung "Walfisch" von den "praktischen Bedürfnissen « bestimmter Berufsgruppen her durchaus zu rechtfertigen (S. 92f.). Die Begriffsbildung aufgrund anatomischer und physiologischer Gegenstandsmerkmale ist - nach Erdmann - nicht per se 'besser' als die Begriffsbildung unter dem Aspekt sozialen Nutzens. Dabei ist nicht zu bestreiten, daß Präzisierungen der Wortbedeutung für bestimmte Bereiche notwendig und sinnvoll sind, für den alltäglichen Gebrauch wären sie jedoch hinderlich, denn hier ist "eine gewisse Vagheit « notwendig (vergleiche Keller, 1995, S. 88).

Ausführlich zeigt Erdmann Probleme der Wortabgrenzung, die sich bei der Interpretation von einfachen, alltagssprachlichen Wörtern wie "Nacht" beziehungsweise "Nachtzeit" in juristischen Texten (zum Beispiel Gesetzesparagraphen) vor allem daraus ergeben, daß das betreffende Wort mehrere wichtige 'Sonderbedeutungen' hat, die miteinander konkurrieren: "Nacht" als astronomischer Begriff für die Zeit zwischen Sonnenuntergang und -aufgang und "Nacht" als sozialer Begriff für die Zeit der ortsüblichen Nachtruhe. Besonders auffällig ist hier, daß beide 'Sonderbedeutungen' unscharfe Begriffe erzeugen: Man kann sowohl darüber streiten, wann die Dämmerung aufhört und die Dunkelheit beginnt (also wo die Grenzen des astronomischen oder astronomisch fundierten Begriffs liegen), als auch darüber, was ortsüblich als Zeit der Nachtruhe anzusehen ist. Erdmann bietet für das Nebeneinander solcher 'Sonderbedeutungen' auch im Kontext von fachindizierten juristischen Zusammenhängen keine normative Lösung an. Stets komme es darauf an, »die Gründe «, die für die eine oder andere Bedeutung sprächen, "gegeneinander abzuwägen “ $(1910$, S. 99).

$\mathrm{Zu}$ erwähnen ist im Kontext vorkognitivistischen Gedankengutes auch die Wortfeldforschung. Sie wird allgemein als Übergangsparadigma von der isolationistischen Semasiologie und Onomasiologie zu einer strukturalistischen Bedeutungskonzeption (mit bilateralem Zeichenbegriff) eingeordnet. Es kann jedoch, besonders bei J. Trier, gezeigt werden, daß eine scharfe Unterscheidung zwischen langue und parole, aber insbesondere zwischen langue und Kompetenz hier fehlt. Die Feldstruktur, die wir heute als Charakteristikum der langue betrachten, faßt er als im Sprachverarbeitungsprozeß notwendigerweise präsente Größe auf. Das Feld hat gegenüber der ungegliederten Bewußtseins- 
substanz gliedernde Funktion, das genaue »Verstehen eines Wortes ist abhängig von der seelischen Gegenwärtigkeit des Gesamtfeldes“ (Trier, 1931, S. 7). Das heißt, Felder oder wortfeldartige Gruppierungen sind offenbar "Bestandteil der kognitiven Ausstattung des Menschen « (Stachowiak, 1979, S. 38) und können daher auch als Organisationsformen des mentalen Lexikons gedeutet und mit dem Kognitivismus in Verbindung gebracht werden, wie zum Beispiel Lutzeier (1995, S. 26f.) dies auch anregt.

So viel zu den historischen Paradigmen, von denen ich drei genannt habe. Aus der historischen Betrachtung, die ja tendenziell die Existenz von zwei konkurrierenden Grundpositionen, die jeweils in den Strukturalismus beziehungsweise Kognitivismus münden, erkennen ließ, ergeben sich nun Fragen zu den modernen Paradigmen, zum Beispiel: Ist der Unterschied zwischen strukturalistischer und kognitivistischer Konzeption in der Tat grundsätzlicher Natur? Hat er Auswirkungen für die lexikologisch-lexikographische Praxis?

Ich möchte deshalb - sozusagen als Zielpunkt der historischen Analyse auch die semantischen Positionen der beiden modernen Paradigmen mit Hilfe der Rasterparameter kurz charakterisieren.

\section{Das strukturalistische Paradigma}

Der Strukturalismus gelangt aufgrund seines bilateralen Zeichenbegriffs zu einer Differenzierung zwischen Wortbedeutung (Signifikat) und Begriff, also im Prinzip zu einer Triade. Das Verhältnis zwischen Bedeutung und Begriff ist jedoch facettenreich und uneinheitlich innerhalb des Strukturalismus: Man kann für das Verhältnis zwischen diesen beiden Kategorien beziehungsweise für die Frage, wie Bedeutungen sich auf Begriffe beziehen, hier schematisch drei Positionen unterscheiden.

\subsection{Das Begriffsinventar als amorpher Stoff}

Unter der ersten Position wird das Begriffs- oder Vorstellungsinventar als ein unzugänglicher amorpher Stoff betrachtet, als gedankliche beziehungsweise mentale Substanz, die erst durch Sprache geformt und strukturiert wird. Sprache ist konstitutiver Faktor der Gedankenformung und -bildung. Dies ist die Position von Humboldt, dann, etwas undeutlich, von Saussure, schließlich klarer bei Hjelmslev.

Gerade die Position Saussures ist, auch bedingt durch die problematische Quellenlage seines Werkes, nach wie vor nicht exakt zu umreißen. So wird Saussure einerseits - vor allem von Jäger $(1976,1985)$ und Stetter $(1985)$ - in die idealistisch-hermeneutische Tradition etwa Humboldts gestellt, derzufolge das sprachliche Zeichen, in der Terminologie der frühen Notes 'item das Sème, synthetischen Charakter hat, insofern als es »Zeichen und Bedeutung vereint in 
einer Art von Persönlichkeit « (Jäger, 1985, S. 15). In diesem synthetischen Zeichen erst kommen die jeweils amorphen Bereiche des Lautlichen und des Bedeutungshaften zu konkreter Individualität. Insofern also setze Saussures Semiologie die anti-repräsentationistische Tradition des Idealismus fort, derzufolge sprachliche Zeichen keineswegs als bloße Nomenklatur vorsprachlicher Begriffe fungierten. Andererseits jedoch eröffnet Saussure durch den Gedanken des "valeur «, also durch die Betonung der systemhaften Bestimmbarkeit und der rein 'innersprachlichen' Konstitution der Zeichen und ihrer Bedeutung, den Weg zu einer Bedeutungskonzeption, bei der die Bezüglichkeit selbst auf einen auch nur amorphen begrifflichen Stoff völlig in den Hintergrund tritt gegenüber den reinen Beziehungen der Sèmes untereinander (vergleiche Abschnitt 4.3).

\subsection{Universales Begriffsinventar}

Der zweiten Position zufolge erscheint das Begriffsinventar als solches universal gültig und aus den Sachen ableitbar. Für jeden Begriff lassen sich notwendige und hinreichende Merkmale unterscheiden. Bedeutungen sind dann als einzelsprachliche Zusammenfassungen jeweils eines spezifischen Komplexes solcher begrifflicher Merkmale zu sehen. Dies ist die Position des amerikanischen Strukturalismus (zum Beispiel Katz \& Fodor, 1963).

\subsection{Lexikalisches Wissen versus enzyklopädisches Wissen}

Bei der dritten, explizitest strukturalistischen Position wird versucht, Bedeutungsanalyse von einer sachbezogenen Analyse weitgehend abzukoppeln. Die Bedeutung soll allein aufgrund von innersprachlichen Beziehungen (also Feldbeziehungen, syntagmatischen Beziehungen) ermittelt werden. Dies ist - in Fortführung des Saussureschen valeur-Gedankens - die Position des europäischen Strukturalismus (zum Beispiel bei Pottier oder Coseriu). Vor allem hier wird die Trennung von lexikalischem und enzyklopädischem Wissen zur substanziellen Frage. Der Strukturalismus kann dabei einen methodisch klaren Standpunkt einnehmen: Lexikalisches Wissen ist alles, was Bedeutungsunterschiede zwischen Wörtern konstituiert und was sinnrelational zu ermitteln ist; alles andere fällt aus der lexikalischen Bedeutung heraus: Da zum Beispiel Gattungsbegriffe (natural kinds) wie "Vogel" oder "Hund" keine Felder konstituieren, ist ihre lexikalische Bedeutung - übertrieben ausgedrückt - nahezu 'leer'.

Hinsichtlich dieses Strukturalismus im engsten Sinne stellt sich jedoch die methodische Frage, woher diese vorgeblich rein innersprachliche, strukturelle Analyse ihren Stoff bezieht, woher die Merkmale innersprachlicher Abgrenzung kommen - sind sie doch letztlich auch in der Substanz begrifflich-sachlicher Natur beziehungsweise stammen sie aus konzeptuellem, begrifflichem 
Wissen. Das heißt, der europäische Strukturalismus scheint das Kind mit dem Bade auszuschütten. Zwar vertritt er zu Recht die Auffassung, Bedeutung und Begriff seien nicht identisch, ebensowenig wie Bedeutungsanalyse und Begriffsanalyse. Er verkennt aber, daß der Gebrauch von Wörtern bestimmter Art eine Kategorisierungsleistung im Hinblick auf die Welt impliziert, also Gebrauchsregeln und Begriffsprägung untrennbar verbunden sind. Insofern ist der Rückgriff strukturalistischer Analysen auf begriffliche Merkmale durchaus vertretbar - nur bekennt der Strukturalismus sich nicht dazu.

\section{Das kognitivistische Paradigma}

Ich komme nun zum letzten, dem kognitivistischen Paradigma: Für den Kognitivismus sind Begriff beziehungsweise Konzept und Bedeutung mentale Einheiten derselben Art. Dies gilt unabhängig davon, ob eine ein- oder zwei- oder gar dreistufige Bedeutungskonzeption angesetzt wird (zum Beispiel Bierwisch \& Lang, 1987; Jackendoff, 1983; Schwarz, 1992, 1995). An die Stelle der languegebundenen Bedeutung tritt das kompetenz-gebundene Konzept (als Einheit des Langzeitgedächtnisses). Konzepte sind - universal geprägte - mentale Repräsentationen, sind 'Konzeptualisierungen' von Entitäten und Ereignissen der außersprachlichen Wirklichkeit. Die Unterscheidung zwischen enzyklopädischem und lexikalischem Wissen wird, wo nicht ganz aufgegeben, doch eher marginalisiert: Lexikalisches Wissen wird zu einer speziellen Form der 'Bindung' oder 'Verpackung' konzeptuellen Wissens, Sprache wird zum Instrument des Ausdrucks unvollständiger konzeptueller Information, die durch das umfassende nicht-sprachlich vermittelte Welt- und Situationswissen zu ergänzen ist. Von daher läßt sich die Frage stellen, ob man innerhalb des Kognitivismus beziehungsweise innerhalb psychologischer Theorien nicht auf eine der drei Kategorien, nämlich den Bedeutungsbegriff, als für die Theoriebildung unnötig verzichten könnte (vergleiche die Beiträge von Hoffmann und von Herrmann, Grabowski, Schweizer \& Graf in diesem Band). Bedeutung wäre dann definiert nur noch als Relationsbegriff, nämlich als Relation zwischen Wort und sprachlich gebundenem, aber der Natur nach mentalem - Begriff, was wiederum allerdings so neu nicht wäre - man denke an Adelung beziehungsweise an die Vertreter des dyadischen Paradigmas.

\section{Teil II Die Paradigmen und die Wörterbuchpraxis am Beispiel von Adelungs "Frucht"-Artikel}

Im zweiten Teil des Aufsatzes soll den theoretischen Abgrenzungen des ersten Teils eine empirische Analyse von Bedeutungsbeschreibungen gegenübergestellt werden, und zwar in Form der lexikographischen Bedeutungserläuterung, wie sie Adelung für Appellativa, insbesondere das Wort "Frucht" vorlegt. Diese 
Konfrontation mit der lexikographischen Praxis kann als Prüfstein, möglicherweise sogar als Korrektiv für die (in Teil I) jeweils behauptete theoretische Position betrachtet werden. Adelung wurde im ersten Teil von seiner theoretischen Position her als Vertreter des klassisch-dyadischen Paradigmas eingeordnet, eine solche Klassifikation kann aber dem Pionier der deutschsprachigen Lexikographie und damit der praktischen Lexikologie insgesamt nicht voll gerecht werden. Wie verfährt er konkret im Hinblick auf Bedeutungsbeschreibung und/oder Begriffsanalyse? Hier ein Ausschnitt aus dem entsprechenden Wortartikel (vergleiche Adelung, 1796):

Die Frucht ...

1. Eigentlich. 1) In der weitesten Bedeutung, alles was die Erde zur Speise für Menschen und Thiere, besonders aber die erstern hervor bringet; ... Feldfrüchte, was von dieser Art auf dem Felde wächset ... Baumfrüchte, was auf Bäumen wächset. Gartenfrüchte, was in Gärten gebauet wird. ... In etwas anderm Verstande verstehet man unter diesem Ausdrucke zuweilen die Samen und Samenbehältnisse aller Pflanzen und Bäume, sie mögen nun dem Thierreiche, und besonders dem Menschen, zur Speise dienen oder nicht, mit Ausschließung der übrigen Theile der Pflanzen.

2) In engerer Bedeutung. (a) Das Getreide, oder die Samen verschiedener Grasarten, welche zur Speise gebraucht werden; ...

(b) Die Frucht einiger Bäume, Obst. ... Wilde Früchte, d. i. wildes Obst. Früchte einmachen, Nüsse, Datteln, Pflaumen u.s.f. Wälsche Früchte, Früchte der Orange-Bäume, Datteln u.s.f.

Konzentriert man sich ausschließlich auf die Bedeutungsangaben des Artikels, so unterscheidet Adelung mehrere, zum Teil hierarchisch ineinander eingebettete Bedeutungen von "Frucht". Die Logik seiner Artikelanlage generell ist die des Fortschreitens von sinnlichen zu abstrakten ('übertragenen') Bedeutungen, wobei häufig die Ansetzung ursprünglicher sinnlicher Bedeutungen äußerst spekulativ und heute unter etymologischer Perspektive nicht nachzuvollziehen ist. Aber dies ist ein anderes Thema. Wir beschränken uns hier im wesentlichen auf die sogenannten 'konkreten' Bedeutungen von "Frucht".

Adelung setzt sieben Varianten für "Frucht" an. Bleiben wir also innerhalb der Struktur seines Paradigmas, so müssen wir annehmen: Für ihn ist "Frucht" Bezeichnung von sieben verschiedenen vorsprachlichen Begriffen, die - dies ist in der Tat so - nach genus proximum und differentia specifica gliedernd beschrieben werden können: "Aufgabe der Beschreibung ist, den Begriff eines jeden Wortes und [den Begriff, G. S.] einer jeden Bedeutung desselben [Wortes, G. S.] möglichst genau zu bestimmen « (Adelung, 1793, S. vi). Die hierarchischen Beziehungen müßten also an gemeinsamen Oberbegriffen unterschiedlicher Stufen ablesbar sein. 
Ich möchte nun folgende Frage stellen: Prägt die theoretische Vorgabe des klassisch-dyadischen Modells die konkrete Analyse unverwechselbar, oder kann die Adelungsche Analyse von "Frucht" auch anders gelesen werden, zum Beispiel im Sinne des Strukturalismus oder der Gebrauchstheorie? Im Hinblick auf diese Frage gibt es von einer Seite bereits Vorschläge. Henne (1972, S. 83ff. und besonders S. 100ff.) re-interpretiert Adelungs Vorgabe - bewußt ahistorisch - als Signifikatbeschreibung eines bilateralen Wortes mit sieben Sememen. Er führt die Adelungsche Bedeutungsbeschreibung zurück auf eine Merkmalsanalyse mit Hilfe von insgesamt zwölf semantischen Merkmalen wie zum Beispiel Produkt, menschlicher/tierischer Bereich, zum Verzehr bestimmt, Speise für Menschen und Tiere, Ware und so weiter (vergleiche Henne, 1972, S. 102). Mit Hilfe dieser Merkmalsmatrix kann Henne über das Vorkommen gemeinsamer Merkmale in verschiedenen Sememen die interne Gliederung des Sememverbandes, die Adelung vornimmt, nachzeichnen.

Die Hennesche "Transformation « (1972, S. 112ff.) wirft ein Schlaglicht auf unser Gesamtproblem, insbesondere auf das Verhältnis von klassisch-dyadischer Begriffsanalyse und Strukturalismus. Unterstellt man - wie ich es tue -, daß Hennes Analyse im großen und ganzen angemessen ist, so würde dies zeigen, daß begriffsanalytische Operationen und semantische Operationen letztlich zu identischen Ergebnissen führen. Dies bedeutet wiederum für das strukturalistische Paradigma, daß in ihm der Bilateralismus überbewertet wurde und $\mathrm{da} ß$ die Betonung von Bilateralität und Sprachimmanenz nur zu einer Reformulierung bereits in anderen Paradigmen erarbeiteter Analysen führt.

Allerdings ist die strukturalistische Reinterpretation mit einer ganzen Reihe von Einschränkungen zu versehen: Henne legt einen merkmalsemantischen Ansatz von notwendigen und hinreichenden Merkmalen, die den bezeichneten Gegenständen zukommen müssen, zugrunde, also eine Merkmalsemantik nach dem Vorbild des amerikanischen Strukturalismus. Damit wird er weder dem Strukturalismus noch Adelung voll gerecht.

Einerseits gelangt Henne zu seiner - beziehungsweise Adelungs - Merkmalsanalyse durch einfache Setzung, ohne die Sememe aus innersprachlichen Relationen abzuleiten. Strukturalistisch strenggenommen ist der Ansatz von sieben Sememen nur zu rechtfertigen, wenn auch sieben Felder oder Oppositionspaare zu konstituieren sind, aus denen sich die Merkmale von "Frucht" ergeben.

In diesem Sinne streng strukturalistisch ist Adelungs Analyse nicht, denn Feldbeziehungen deutet er nur an (zum Beispiel durch den Verweis auf Ausdrücke wie "Obst", "Getreide", "Samen"). Und es ist anzunehmen, daß eine striktere innersprachliche Relationsanalyse zumindest zu einer anderen Ordnung der Sememe, eventuell zu einer anderen Aufgliederung insgesamt geführt hätte.

Andererseits läßt Adelungs Analyse Spielräume zu, die eine strikte Merkmalsanalyse nicht erlaubt. Damit bin ich bereits bei einer zweiten möglichen Reinterpretation, zu der ich durch die Lektüre von Kellers Zeichentheorie 
(1995) angeregt wurde. Das Wort "Frucht" erzeugt, wie Keller sagen würde, offensichtlich in seinem gemeinsprachlichen Gebrauch einen Begriff mit unscharfen Rändern und möglicherweise auch einer Prototypenstruktur. Zweifellos sind Äpfel, Birnen, Mangos Früchte, aber sind Kartoffeln, Zwiebeln, Karotten Früchte gleichen Rechts? Kompliziert wird der Gebrauch von "Frucht" noch dadurch, daß fachsprachlich der Begriff der Frucht ein klar definierter, scharfer taxonomischer Begriff ist, wie zum Beispiel Fachwörterbücher bestätigen:

Frucht Bot., der infolge der Befruchtung umgewandelte Fruchtknoten. Die Wand des letzteren wird zum Fruchtgehäuse, welche den aus d. Samenknospe hervorgegangenen Samen einschließt. Je nach d. Zahl d. Samen, welche sie einschließt, heißt d. Frucht einod. mehrsamig. ... Unter echter Frucht versteht man den vergröBerten und in seiner Beschaffenheit veränderten Fruchtknoten in d. Zustande, wo er reife Samen enthält “ (Bechhold, 1919, S. 513).

Erfaßt Adelung diesen komplexen (alltags- und fachsprachlichen) Gebrauch von "Frucht" nicht recht gut? Mit der Erläuterung von Bedeutung 1.1) beschreibt er die vage allgemeinsprachliche Bedeutung: "alles was die Erde zur Speise für Menschen und Thiere ... hervor bringet «. Adelung gibt hier also den Nutzungsaspekt als Kriterium an und spezifiziert damit - durchaus angemessen - einen Begriff mit unscharfen Rändern.

Diesem allgemeinsprachlichen vagen Gebrauch stellt er (noch innerhalb von Bedeutung 1.1) den fachsprachlichen zur Seite. Der fachsprachliche Gebrauch hebt allein auf biologisch überprüfbare Objektmerkmale ab und erzeugt daher - nach Keller - einen scharfen Fregeschen Begriff. Der Nützlichkeitsaspekt fält hier notwendigerweise weg, Adelung weist ausdrücklich darauf hin.

Mit der Angabe von Bedeutung 2b): "die Frucht einiger Bäume, Obst “ deutet Adelung an, daß es offensichtlich bessere Vertreter der Kategorie Frucht gibt und weniger gute: Äpfel, Birnen, Obst als prototypische Vertreter der Kategorie Frucht.

Die verschiedenen Verwendungsweisen von "Frucht" überschneiden sich, ohne sich zu decken: Nicht alles, was die Erde zur Speise hervorbringt, ist im fachsprachlichen Sinne Frucht (zum Beispiel ist die Kartoffel eine Knolle, die Karotte eine Wurzel). Umgekehrt ist nicht alles, was im fachsprachlichen Sinne Frucht ist, auch eßbar (zum Beispiel Tannenzapfen).

Man kann also folgendes Fazit ziehen: Trotz des monolateralen Zeichenbegriffs beschreibt Adelung die Gebrauchsregeln von Wörtern, nicht sprachunabhängige Begriffe. Die mangelnde Reflexion über das Verhältnis von Sprache und Begriff - Begriffe sind für Adelung ja präexistent, die sprachliche Einheit repräsentiert nur den Begriff - steht einer gebrauchsregelbeschreibenden Praxis nicht im Wege (so daß man sagen kann: Adelung ist in seiner Praxis weiter als in seiner Theorie!). Daß Adelung sich Zugang zu dem, was er für Begriffe hielt, nur über eine genaue Bedeutungsanalyse verschaffte, ist offensichtlich. 
Diese Tatsache der Übergänglichkeit zwischen Begriffsanalyse und $\mathrm{Ge}$ brauchsanalyse nutze ich in einer Zwischenbemerkung zu einem kurzen Ausblick auf die Gebrauchstheorie: Die Gebrauchstheorie der Bedeutung wurde im ersten Teil des Aufsatzes als poststrukturalistisch eingeordnet. Sie teilt mit dem Strukturalismus die Überzeugung, daß Bedeutungen sich im Raum einer Einzelsprache als soziale Tatsache sprachlich konstituieren, nicht begrifflich, und daß daher ein Zugang zu Bedeutungen nur über Sprachanalyse zu gewinnen ist. Die Gebrauchstheorie geht über den Strukturalismus insofern hinaus, als sie Sprache nicht als statisch-abstraktes System von Einheiten faßt, sondern als instrumentales System von Regeln, die die Sprecher einer Sprache zum Zweck der Verständigung als Teil ihrer Lebenspraxis im Gebrauch jeweils neu anwenden und modifizierend realisieren. Die Gebrauchstheorie versteht sich als nicht-repräsentationistisch, sie weist also eine direkte Rückführung von Bedeutungen auf außersprachliche, somit auch begriffliche Entitäten zurück. Was repräsentationistische Theorien als Begriff, zum Beispiel den oder einen der Begriff(e) Frucht, rekonstruieren, entspricht gebrauchstheoretisch einer Gebrauchsregel, die besagt, daß wir nur Gegenständen bestimmter Art mit Wahrheit das Charakteristikum Frucht zuschreiben können. Klassifikationen sind somit Resultate sprachlicher Praxis, Begriffe bilden sich im Zusammenhang mit und aufgrund des Gebrauchs von Wörtern.

Zurückkehrend zu Adelung bestätigt dieser Exkurs unsere Hypothese: Eine sorgfältige Analyse des Sprachgebrauchs, wie Adelung sie zumindest anvisiert, macht uns in erster Linie die Gebrauchsregeln eines Wortes deutlich, bei Appellativa wie "Frucht" erschließt sie darüber hinaus das Netzwerk von Klassifikationen, die dieses Wort bereitstellt, und somit den Bestand an sprachbasierter begrifflicher Kategorisierung. Wenn Adelung Begriffe (gegenüber der Sprache) für vorgängig hielt, so ist dies eine - von der Theorie insinuierte perspektivische Verschiebung.

Wir können also verallgemeinernd festhalten: Adelungs Lexikoneintrag als relativ angemessene Analyse der Bedeutung von "Frucht" läßt - je nach Perspektive - unterschiedliche theoriegebundene Lesarten zu, nämlich eine im ursprünglichen klassisch-dyadischen Sinne (das Wort "Frucht" repräsentiert mehrere verschiedene außersprachliche Begriffe), eine strukturalistische und eine im Sinne der Gebrauchstheorie.

Sollte uns diese Erkenntnis nicht dazu veranlassen, den Stellenwert strenger theoretischer Abgrenzungen zwischen Bedeutung einerseits und Begriff andererseits zu relativieren? 


\section{Teil III Anhang: Ein Raster zur Relation von Wort - Bedeutung - Begriff}

(1) Zeichenauffassung:

(1a) Monolateralität des Zeichens versus

(1b) Bilateralität des Zeichens.

Wenn (la), dann gilt:

(2) Wörter bezeichnen/bedeuten Sachen und ähnliches oder

(3) Wörter bezeichnen/bedeuten außer(einzel)sprachliche Begriffe.

(2) Dies ist, vergröbert, die Position der Wörter-und-Sachen-Forschung und wohl auch der vorstrukturalistischen Semasiologie und Onomasiologie. Nur bei Schuchardt wird 'Vorstellung' als Vermittlungsinstanz angesetzt. Ermöglicht wird diese - was die Reflexion über Bedeutungen angeht, relativ überholte - Position durch die Konzeption, Sprachgeschichte als Teil der Kulturgeschichte zu betrachten, durch die Konzentration auf die Geschichte der 'Sachen' und den Wandel ihrer Bezeichnungen. Ansätze zu einer komplexeren, bilateralen Auffassung zeigen sich besonders bei Schuchardt, bei ihr werden vier (beziehungsweise fünf) verschiedene Größen unterschieden; dies haben Wiegand und Harras (1971) herausgearbeitet.

(3a) Begriffe sind Allgemeinbegriffe (im Sinne des Realismus). Dies ist die abbildtheoretische Position: Sachverhalte der bewußtseinsunabhängigen Welt werden so erkannt, wie sie sind (Frege, Carnap, Husserl). Diese Position spielt für die weitere Erörterung keine Rolle.

(3b) Begriffe sind individualpsychologische Vorstellungen (Nominalismus). Dies ist, vergröbert, die Position H. Pauls.

(3c) Begriffe/Konzepte sind universal geprägt durch angeborene, artspezifische Erkenntniskategorien beziehungsweise kognitive Kategorien (dies ist der universalistisch-anthropologische Idealismus): Das menschliche Erkenntnissystem bestimmt die Weltsicht.

Dies ist die Kantsche Position, sie gilt für die frühen Lexikographen des 18. Jahrhunderts und für viele Forscher des 19. Jahrhunderts (Reisig und andere). Der moderne Kognitivismus führt diese Position fort. Er nimmt artbezogene Universalien an, die die Konzeptausbildung steuern. Im modernen Kognitivismus, der sich als psychologische Theorie versteht und psychologische empirische Methoden anwendet, gibt es aber auch Verbindungen zu ( $3 b)$, insofern Konzepte, obwohl universal determiniert, doch als individual-mentale Einheiten begriffen werden. 
Wenn (lb), dann gilt:

(4) Wörter haben ein an sie gebundenes Signifikat oder Bedeutung(en).

(4a) Bedeutung wird als Bindung bestimmter sachbezogener Begriffe oder Begriffskombinationen an einen einzelsprachlichen Wortkörper gefaßt. Diese Position läßt im Prinzip für die Begriffe selbst wieder die Positionen $(3 a)$ bis $(3 c)$ zu. Man kann unterscheiden:

(4a.1) Das Begriffsinventar ist ein unzugänglicher, amorpher Stoff, die Substanz, die die Sprache formt und strukturiert. Sprache ist konstitutiver Faktor der Gedankenformung und -bildung (Humboldt teilweise, Saussure, Hjelmslev).

(4a.2) Das Begriffsinventar ist universal, für jeden Begriff lassen sich notwendige und hinreichende Merkmale unterscheiden, die aus den Sachen abgeleitet sind. Dies ist die Position des amerikanischen Strukturalismus.

(4a.3) Die Konzepte sind (weitgehend) universal, sie folgen jedoch Prototypizitätsprinzipien. Das heißt, nicht alle Merkmale sind jeweils notwendig (moderne kognitive Pototypentheorie; vergleiche Blutner, 1995).

(4a.4) Die Strukturierung der Welt ist einzelsprachlich, nicht-universal (vergleiche den einzelsprachlichen Idealismus, die sogenannte Relativitätstheorie), besonders Humboldt, Weisgerber ("sprachliches Weltbild«).

(4b) Bedeutung wird von sachbezogenen Begriffen weitgehend abgekoppelt. Sie soll in innersprachlichen Beziehungen (Feldbeziehungen, syntagmatischen Beziehungen) eruierbar sein. Dies ist die Position des europäischen Strukturalismus, besonders Coseriu.

(5) Unterscheidung zwischen lexikalischem und enzyklopädischem Wissen

(5a) wird getroffen oder

$(5 b)$ wird nicht getroffen.

(5a) ist nur bei (4) möglich, nicht bei (3).

Alle Positionen von (4a) haben jedoch Schwierigkeiten mit dieser Unterscheidung, das heißt, sie können keine klaren Grenzen angeben zwischen lexikalischem und enzyklopädischem Wissen. Nur (4b) behauptet, dies zu können.

(6) Die Unterscheidung zwischen langue und parole wird

(6a) gemacht,

(6b) nicht gemacht, oder

(6c) als Unterscheidung zwischen Kompetenz und Performanz aufgefaßt. 
(6a) trifft für die strukturalistische Position zu;

(6b) wohl für fast alle vorstrukturalistischen Positionen;

(6c) für den Kognitivismus und ansatzweise H. Paul. Beim modernen Kognitivismus repräsentiert das Langzeitgedächtnis beziehungsweise das dort gespeicherte mentale Lexikon die Kompetenz, also die Sprachfähigkeit, den abstrakten Sprachbesitz (vergleiche 'System'), das Kurzzeitgedächtnis repräsentiert die Anwendung dieser Kompetenz in der Sprachtätigkeit und -verarbeitung (Performanz).

\section{Literatur}

Abel, C. (1878). Die englischen Verba des Befehls. Berlin: Liepmannssohn.

Abel, C. (1882). On philological methods. In C. Abel, Linguistic essays (S. 157-167). London: Trübner.

Adelung, J. C. (1789). Ueber den deutschen Styl. Erster Band (dritte vermehrte und verbesserte Auflage). Berlin: Voß.

Adelung, J. C. (1793). Auszug aus dem grammatisch-kritischen Wörterbuche der Hochdeutschen Mundart. Erster Theil, von A - E. Leipzig: Breitkopf.

Adelung, J. C. (1796). Auszug aus dem grammatisch-kritischen Wörterbuche der Hochdeutschen Mundart. Zweyter Theil, von F - L. Leipzig: Breitkopf.

Bechhold, J. H. (Hrsg.). (1919). Handlexikon der Naturwissenschaften und Medizin (2. Auflage). Frankfurt/M.: Bechhold.

Bierwisch, M. (1983). Semantische und konzeptuelle Repräsentation lexikalischer Einheiten. In R. Ruzicka \& W. Motsch (Hrsg.), Untersuchungen zur Semantik (S. 611-699). Berlin: AkademieVerlag.

Bierwisch, M. \& Lang, E. (Hrsg.). (1987). Grammatische und konzeptuelle Aspekte von Dimensionsadjektiven. Berlin: Akademie-Verlag.

Blutner, R. (1995). Prototypen und Kognitive Semantik. In G. Harras (Hrsg.), Die Ordnung der Wörter (S. 227-270). Berlin: de Gruyter.

Campe, J. H. (1808). Wörterbuch der Deutschen Sprache. Braunschweig: Schulbuchhandlung.

Carnap, R. (1942). Introduction to semantics. Cambridge, MA: MIT Press.

Coseriu, E. (1967). Lexikalische Solidaritäten. Poetica, 1, 293-303.

Coseriu, E. (1972). Die Geschichte der Sprachphilosophie von der Antike bis zur Gegenwart. Eine Ubersicht. Teil II: Von Leibniz bis Rousseau. Vorlesungen, gehalten im Winter-Semester 1970/71 an der Universität Tübingen. Autorisierte Nachschrift. Tübingen: Narr.

Coseriu, E. (1992). Strukturelle und kognitive Semantik. Vorlesung, gehalten im Winter-Semester 1989/90. Nachschrift von U. Maier und H. Weber. Tübingen.

Coseriu, E. \& Geckeler, H. (1981). Trends in structural semantics. Tübingen: Narr.

Dieckmann, W. (1981). K. O. Erdmann und die Gebrauchsweisen des Ausdrucks "Konnotationen" in der linguistischen Literatur. In W. Dieckmann (Hrsg.), Politische Sprache. Politische Kommunikation. Vorträge. Aufsätze. Entwürfe (S. 78-136). Heidelberg: Winter.

Eberhard, J. H. (1795). Versuch einer allgemeinen deutschen Synonymik in einem historisch-philosophischen Wörterbuche der sinnverwandten Wörter der hochdeutschen Mundart. Erster Theil. A C. Nebst einem Versuch einer Theorie der Synonymik. Halle/Leipzig: Ruff.

Erdmann, K. O. (1910). Die Bedeutung des Wortes. Aufsätze aus dem Grenzgebiet der Sprachpsychologie und Logik (2. Auflage). Leipzig: Avenarius.

Frege, G. (1892). Über Sinn und Bedeutung. Zeitschrift für Philosophie und philosophische Kritik, $100,25-50$. 
Haase, F. (1874-1880). Vorlesungen über lateinische Sprachwissenschaft. 2 Bände. Band I: Einleitung, Bedeutungslehre (herausgegeben von F. A. Eckstein, 1874). Band II: Bedeutungslehre (Zweiter Theil) (herausgegeben von H. Peter, 1880). Leipzig: Simmel.

Harras, G. (in diesem Band). Zwischen Intentionalität und Konvention: Bedeutungskonzepte für kommunikative Handlungen (S. 67-87).

Hecht, M. (1888). Die griechische Bedeutungslehre, eine Aufgabe der classischen Philologie. Leipzig: Teubner.

Heerdegen, F. (1875-1881). Untersuchungen zur lateinischen Semasiologie. Erlangen: Deichert.

Heerdegen, F. (1890). Grundzüge der lateinischen Bedeutungslehre. In Ch. K. Reisig, 1881-1890, Band II (S. 39-154).

Henne, H. (1972). Semantik und Lexikographie. Untersuchungen zur lexikalischen Kodifikation der deutschen Sprache (= Studia Linguistica Germanica 7). Berlin: de Gruyter.

Herrmann, Th., Grabowski, J., Schweizer, K. \& Graf, R. (in diesem Band). Die mentale Repräsentation von Konzepten, Wörtern und Figuren (S. 120-152).

Heyse, K. W. L. (1856). System der Sprachwissenschaft (herausgegeben von H. Steinthal). Berlin: Dümmler.

Hjelmslev, L. (1972). Prolegomena zu einer Sprachtheorie (übersetzt von R. Keller, U. Scharf \& G. Stötzel). München: Hueber.

Hoffmann, J. (in diesem Band). Die Genese von Begriffen, Bedeutungen und Wörtern (S. 88-119).

Humboldt, W. von (1903-1936). Wilhelm von Humboldts Gesammelte Schriften. Herausgegeben von der Königlich-Preussischen Akademie der Wissenschaften. Berlin. 17 Bände. [Nachdruck 1968. Berlin: de Gruyter.]

Husserl, E. (1901). Logische Untersuchungen II (5. Auflage 1968). Tübingen: Niemeyer.)

Jackendoff, R. S. (1983). Semantics and cognition. Cambridge, MA: MIT Press.

Jäger, L. (1976). F. de Saussures historisch-hermeneutische Idee der Sprache. Ein Plädoyer für die Rekonstruktion des Saussureschen Denkens in seiner authentischen Gestalt. Linguistik und Didaktik, 27, 210-244.

Jäger, L. (1985). Der saussuresche Begriff der Aposeme als Grundlagenbegriff einer hermeneutischen Semiologie. In L. Jäger \& C. Stetter (Hrsg.), Zeichen und Verstehen. Akten des Aachener Saussure-Kolloquiums 1983 (S. 7-33). Aachen: Rader.

Jäger, L. (1987). Die Sprachtheorie Wilhelm von Humboldts. In R. Wimmer (Hrsg.), Sprachtheorie. Der Sprachbegriff in Wissenschaft und Alltag (= Jahrbuch 1986 des Instituts für deutsche Sprache) (S. 175-190). Düsseldorf: Schwann.

Johnson-Laird, P. N. (1987). The mental representation of the meaning of words. Cognition, 25, 189-212.

Kant, I. (1781). Kritik der reinen Vernunft (Kants Werke in zehn Bänden. Band III. Herausgegeben von W. Weischedel) (4. Auflage). Darmstadt: Wissenschaftliche Buchgesellschaft.

Katz, J. J. \& Fodor, J. A. (1963). The structure of a semantic theory. Language, 39, 170-210.

Keller, R. (1990). Sprachwandel. Von der unsichtbaren Hand in der Sprache. Tübingen: Francke.

Keller, R. (1995). Zeichentheorie. Zu einer Theorie des semiotischen Wissens. Tübingen: Francke.

Keller, R. (in diesem Band). Begriff und Bedeutung (S. 47-66).

Lutzeier, R. P. (1995). Lexikalische Felder - was sie waren, was sie sind und was sie sein könnten. In G. Harras (Hrsg.), Die Ordnung der Wörter (S. 4-29). Berlin: de Gruyter.

Marty, A. (1926). Uber Wert und Methode einer allgemeinen beschreibenden Bedeutungslehre. Aus dem Nachlaß herausgegeben von $O$. Funke. Reichenberg in Böhmen: Gebrüder Stiepel. (2. Auflage 1950. Bern: Francke.)

Marty, A. (1940). Psyche und Sprachstruktur. Aus dem Nachlaß herausgegeben von O. Funke. Bern: Francke.

Paul, H. (1880). Prinzipien der Sprachgeschichte (9., unveränderte Auflage 1975). Tübingen: Niemeyer. 
Paul, H. (1895). Ueber die Aufgaben der wissenschaftlichen Lexikographie mit besonderer Rücksicht auf das deutsche Wörterbuch. Sitzungsberichte der philosophisch-philologischen und der historischen Classe der k. b. Akademie der Wissenschaften zu München, Jahrgang 1894, 53-91.

Polenz, P. von (1994). Deutsche Sprachgeschichte vom Spätmittelalter bis zur Neuzeit. Band II: 17. und 18. Jahrhundert. Berlin: de Gruyter.

Pottier, B. (1964). Vers une sémantique moderne. Travaux de Linguistique et de Littérature, 2.1, $107-137$.

Reisig, Ch. K. (1839). Vorlesungen über lateinische Sprachwissenschaft (herausgegeben mit Anmerkungen von F. Haase). Leipzig: Lehnhold'sche Buchhandlung.

Reisig, Ch. K. (1881-1890). Vorlesungen über lateinische Sprachwissenschaft (mit den Anmerkungen von F. Haase). 3 Bände. Band I: Etymologie (neu bearbeitet von H. Hagen, 1881). Band II: Lateinische Semasiologie oder Bedeutungslehre (neu bearbeitet von F. Heerdegen, 1890). Band III: Lateinische Syntax (neu bearbeitet von J. H. Schmalz und G. Landgraf, 1888). Berlin: Calvary.

Saussure, F. de (1967-1974). Cours de linguistique générale (édition critique par R. Engler). Wiesbaden: Harrassowitz.

Saussure, F. de (1972). Cours de linguistique générale (édition critique préparée par T. de Mauro). Paris: Payot.

Schmitter, P. (1987). Das sprachliche Zeichen. Studien zur Zeichen- und Bedeutungstheorie in der griechischen Antike sowie im 19. und 20. Jahrhundert. Münster: MAKS Publikationen.

Schuchardt, H. (1905). Sachen und Wörter. Zeitschrift für romanische Philologie, 29, 620-622.

Schuchardt, H. (1912). Sachen und Wörter. Anthropos, 7, 827-839.

Schwarz, M. (1992). Kognitive Semantiktheorie und neuropsychologische Realität. Repräsentationale und prozedurale Aspekte der semantischen Kompetenz. Tübingen: Niemeyer.

Schwarz., M. (1995). Kognitivismus und Lexikon. In G. Harras (Hrsg.), Die Ordnung der Wörter (S. 359-367). Berlin: de Gruyter.

Stachowiak, F. J. (1979). Zur semantischen Struktur des subjektiven Lexikons. München: Fink.

Stachowiak, F. J. (1982). Haben Wortbedeutungen eine gesonderte mentale Repräsentation gegenüber dem Weltwissen? Neurolinguistische Überlegungen. Linguistische Berichte, 79, 12-29.

Stetter, C. (1985). Linguistische Konsequenzen der Semiologie Saussures. In L. Jäger \& C. Stetter (Hrsg.), Zeichen und Verstehen. Akten des Aachener Saussure-Kolloquiums 1983 (S. 35-51). Aachen: Rader.

Trabant, J. (1976). Elemente der Semiotik. München: Beck.

Trabant, J. (1983). Ideelle Bezeichnung. Steinthals Humboldt-Kritik. In A. Eschbach \& J. Trabant (Hrsg.), History of semiotics (S. 251 - 276). Amsterdam: Benjamins.

Trier, J. (1931). Der deutsche Wortschatz im Sinnbezirk des Verstandes. Zur Geschichte eines sprachlichen Feldes. I: Von den Anfängen bis zum Beginn des 13. Jahrhunderts. Heidelberg: Winter.

Weisgerber, L. (1927). Die Bedeutungslehre - ein Irrweg der Sprachwissenschaft? In L. Antal (Hrsg.) (1972), Aspekte der Semantik. Zu ihrer Theorie und Geschichte (S. 53-76). Frankfurt/M.: Athenäum.

Wellander, E. (1917-1928). Studien zum Bedeutungswandel im Deutschen. 3 Bände. Uppsala: Lundequistska.

Wiegand, H. E. (1970). Synchronische Onomasiologie und Semasiologie. Kombinierte Methoden zur Strukturierung der Lexik. Germanistische Linguistik, 3, 243-384.

Wiegand, H. E. \& Harras, G. (1971). Deutscher Wortatlas (= Germanistische Linguistik 1-2). Hildesheim: Olms.

Wundt, W. (1900). Völkerpsychologie. Band I: Die Sprache. Leipzig: Engelmann. 